\title{
ON A CONJECTURE OF BALOG
}

\author{
ADOLF HILDEBRAND
}

\begin{abstract}
A conjecture of A. Balog is proved which gives a sufficient condition on a set $A$ of positive integers such that $A \cap(A+1)$ is infinite. A consequence of this result is that, for every $\varepsilon>0$, there are infinitely many integers $n$ such that both $n$ and $n+1$ have a prime factor $>n^{1-\varepsilon}$.
\end{abstract}

1. Introduction. Some of the most difficult and seemingly unattackable problems in number theory deal with simultaneous properties of integers $n$ and their translates $n+t$, where $t \in \mathbb{N}$ is fixed. The twin prime conjecture, for example, asserts that $n$ and $n+2$ are prime infinitely often.

Another problem of this type, posed by Erdös several times (see e.g. [3]), is to show that, for every fixed $\varepsilon>0$, there are infinitely many integers $n$ such that both $n$ and $n+1$ have a prime factor $>n^{1-\varepsilon}$. In other words, putting

$$
Q_{\alpha}=\left\{n \in \mathbb{N}: P(n)>n^{\alpha}\right\},
$$

where $P(n)$ denotes the largest prime factor of $n$, the conjecture asserts that $Q_{\alpha} \cap\left(Q_{\alpha}+1\right)$ is infinite for every $\alpha<1$.

At the Oberwolfach meeting on analytic number theory in 1982, A. Balog proposed a general conjecture, which gives a sufficient condition on a set $A \subset \mathbb{N}$, such that $A \cap(A+1)$ contains infinitely many elements. To this end, he introduced the concept of " $k$-stability". A set $A \subset \mathbb{N}$ is called $k$-stable if

$$
k A \subset A, \quad k^{-1}(A \cap k \mathbb{N}) \subset A,
$$

where $\lambda A$ denotes the set $\{\lambda a: a \in A\}$, and $A \subset B$ means that $A$ is contained in $B$ up to a set of density zero, i.e., $\mathrm{d}(A \backslash B)=0$. Here and in the sequel, $\mathrm{d}(\cdot)$ denotes the asymptotic density, defined by

$$
\mathrm{d}(A)=\lim _{x \rightarrow \infty} \frac{1}{x} \sum_{\substack{n \leq x \\ n \in A}} 1
$$

(provided this limit exists), and the lower and upper densities $\underline{\mathrm{d}}(\cdot)$ and $\overline{\mathrm{d}}(\cdot)$ are defined analogously by taking the limit inferior and the limit superior, respectively.

Balog [1] showed by an elementary argument that $A \cap(A+1)$ is infinite whenever $A$ is 2 -stable and $\mathrm{d}(A)>1 / 3$, and he made the following

Conjecture (BAlog [1]). If $A \subset \mathbb{N}$ is $p$-stable for every prime $p$ and has positive density, then $A \cap(A+1)$ is infinite.

Received by the editors December 14, 1984 and, in revised form, February 4, 1985.

1980 Mathematics Subject Classification. Primary 10A50, 10L99; Secondary 10H15. 
The sets $Q_{\alpha}, \alpha<1$, introduced above, have positive density (see e.g. [2]), and it is easy to see that they are $k$-stable for every $k \in \mathbb{N}$. Thus Balog's conjecture implies the above-mentioned conjecture that $P(n)>n^{1-\varepsilon}$ and $P(n+1)>(n+1)^{1-\varepsilon}$ holds infinitely often for every fixed $\varepsilon>0$.

The purpose of this paper is to prove Balog's conjecture in a more general form.

2. Results. Given a set $A \subset \mathbb{N}$, we define, for $N \in \mathbb{N}$,

$$
A_{N}=\bigcup_{n, d=1}^{N} \frac{n}{d}(A \cap d \mathbb{N})
$$

These sets form an ascending chain starting with $A_{1}=A$.

TheOREM. If $\mathrm{d}(A)>0$, then $\mathrm{d}\left(A_{N} \cap\left(A_{N}+1\right)\right)>0$ for all sufficiently large $N$. More precisely, for every $\varepsilon>0$ there exist $N(\varepsilon) \in \mathbb{N}$ and $\delta(\varepsilon)>0$ such that $\underline{\mathrm{d}}(A) \geqslant \varepsilon$ implies

$$
\mathrm{d}\left(A_{N} \cap\left(A_{N}+1\right)\right) \geqslant \delta(\varepsilon) \quad(N \geqslant N(\varepsilon)) .
$$

If $A$ is $p$-stable for every prime $p \leqslant N$, then $A$ is $k$-stable for all $k \leqslant N$, so that

$$
A_{N}=\bigcup_{n, d=1}^{N} \frac{n}{d}(A \cap d \mathbb{N}) \subset A \subset A_{N},
$$

and, therefore, $\mathrm{d}\left(A_{N} \cap\left(A_{N}+1\right)\right)=\mathrm{d}(A \cap(A+1))$. Thus the theorem implies Balog's conjecture in the following form.

Corollary 1. If $A \subset \mathbb{N}$ satisfies $\mathrm{d}(A) \geqslant \varepsilon$ and is p-stable for every prime $p \leqslant N(\varepsilon)$, then $\mathrm{d}(A \cap(A+1)) \geqslant \delta(\varepsilon)$ holds, where $N(\varepsilon)$ and $\delta(\varepsilon)$ are as in the Theorem.

Applying this result to the sets $Q_{\alpha} \backslash Q_{\beta}, 0 \leqslant \alpha<\beta \leqslant 1$, we obtain the conjecture mentioned in the introduction in the following slightly more general form.

COROLlaRy 2. Let $0 \leqslant \alpha<\beta \leqslant 1$. Then the set of integers $n$ for which $n^{\alpha}<P(n)$ $\leqslant n^{\beta},(n+1)^{\alpha}<P(n+1) \leqslant(n+1)^{\beta}$ holds has positive lower density.

\section{Lemmas.}

LEMMA 1. For every $k \geqslant 2$ there exist positive integers $n_{1}<\cdots<n_{k}$ satisfying

$$
n_{j}-n_{i}=\left(n_{i}, n_{j}\right) \quad(1 \leqslant i<j \leqslant k) .
$$

Proof. ${ }^{1}$ We define an auxiliary sequence $\left(N_{k}\right)_{k \geqslant 1}$ recursively by

$$
N_{1}=1, \quad N_{k+1}=2 \prod_{i=1}^{k}\left(\sum_{j=i}^{k} N_{j}\right) \quad(k \geqslant 1) \text {. }
$$

By construction,

$$
\sum_{h=i}^{j-1} N_{h}\left|N_{j}\right| N_{j+1}|\cdots| N_{k} \quad(1 \leqslant i<j \leqslant k) .
$$

\footnotetext{
${ }^{1}$ Heath-Brown [4] proved a stronger form of the lemma, where the $n_{i}$ were required to satisfy an additional condition besides (1). Since this additional condition is of no relevance here and complicates the proof considerably, we preferred to give a short proof of the lemma in the form stated.
} 
Thus, if for given $k \geqslant 2$ we put

$$
n_{k}=N_{k}, \quad n_{i}=N_{k}-\sum_{j=i}^{k-1} N_{j} \quad(1 \leqslant i \leqslant k-1),
$$

we have $n_{k}>\cdots>n_{1} \geqslant N_{k} / 2 \geqslant 1$, and

$$
n_{j}-n_{i}=\sum_{h=i}^{j-1} N_{h} \mid N_{k}-\sum_{h=j}^{k-1} N_{h}=n_{j} \quad(1 \leqslant i<j \leqslant k),
$$

which is equivalent to (1).

LEMMA 2. Let $r$ be a positive integer, and for $D \geqslant 1$ let $\mathscr{D}=\mathscr{D}(D, r)$ be the set of positive integers $d \leqslant D$ of the form

$$
d=d_{1} p, \quad(p, r)=1, \quad q\left|d_{1} \Rightarrow q\right| r,
$$

where $p$ and $q$ denote primes. Then we have

$$
\frac{1}{r} \sum_{d \in \mathscr{D}} \frac{1}{d}=\frac{\log \log (D+2)}{\varphi(r)}+O(1)
$$

and

$$
\mathrm{d}\left(\mathbb{N} \backslash \bigcup_{d \in \mathscr{D}} d(r \mathbb{N}-1)\right) \ll \frac{\varphi(r)}{\log \log (D+2)},
$$

where $\varphi$ is the Euler function and the implied constants are absolute.

Proof. Letting $d_{1}$ be an integer all of whose prime factors divide $r$, we have

$$
\begin{aligned}
\sum_{d \in \mathscr{D}} \frac{1}{d} & \leqslant \sum_{d_{1} \leqslant D} \frac{1}{d_{1}} \sum_{p \leqslant D} \frac{1}{p} \leqslant \prod_{p \mid r}\left(1-\frac{1}{p}\right)^{-1} \sum_{p \leqslant D} \frac{1}{p} \\
& =\frac{r}{\varphi(r)}(\log \log (D+2)+O(1))
\end{aligned}
$$

and

$$
\begin{aligned}
\sum_{d \in \mathscr{D}} \frac{1}{d} & \geqslant \sum_{d_{1} \leqslant \sqrt{D}} \frac{1}{d_{1}} \sum_{\substack{p \leqslant \sqrt{D} \\
p+r}} \frac{1}{p} \\
& \geqslant\left(\frac{r}{\varphi(r)}-\sum_{d_{1}>\sqrt{D}} \frac{1}{d_{1}}\right) \log \log (D+2)+O(r) .
\end{aligned}
$$

This yields (3), since

$$
\begin{aligned}
\sum_{d_{1}>\sqrt{D}} \frac{1}{d_{1}} & \leqslant D^{-1 / 4} \sum d_{1}^{-1 / 2}=D^{-1 / 4} \prod_{p \mid r}\left(1-p^{-1 / 2}\right)^{-1} \\
& \ll D^{-1 / 4} \exp \left(2 \sum_{p \mid r} p^{-1 / 2}\right) \ll r D^{-1 / 4} .
\end{aligned}
$$

For the proof of (4) we may suppose

$$
\log \log D \geqslant C \varphi(r),
$$


where $C$ is an arbitrary, but fixed, positive constant. Set $S=\cup_{d \in \mathscr{D}} d(r \mathbb{N}-1)$ and define

$$
f(n)=\sum_{\substack{d \mid n, d \in \mathscr{D} \\ n / d \equiv-1 \bmod r}} 1
$$

Thus $f(n) \geqslant 0$ for all $n \in \mathbb{N}$, and $f(n)>0$ if and only if $n \in S$. We use a variance argument to obtain the desired upper bound for $\mathrm{d}(\mathbb{N} \backslash S)$.

Putting

$$
M=\lim _{x \rightarrow \infty} \frac{1}{x} \sum_{n \leqslant x} f(n)=\sum_{d \in \mathscr{D}} \frac{1}{d} \lim _{x \rightarrow \infty} \frac{d}{x} \sum_{\substack{n \leqslant x / d \\ n \equiv-1 \text { mod } r}} 1=\frac{1}{r} \sum_{d \in \mathscr{D}} \frac{1}{d},
$$

we have

$$
\begin{aligned}
\mathrm{d}(\mathbb{N} \backslash S) & =\lim _{x \rightarrow \infty} \frac{1}{x} \sum_{\substack{n \leqslant x \\
f(n)=0}} 1 \leqslant \lim _{x \rightarrow \infty} \frac{1}{x} \sum_{\substack{n \leqslant x \\
|f(n)-M| \geqslant M / 2}} 1 \\
& \leqslant \frac{4}{M^{2}} \lim _{x \rightarrow \infty} \frac{1}{x} \sum_{n \leqslant x}(f(n)-M)^{2}=\frac{4}{M^{2}}\left\{\lim _{x \rightarrow \infty} \frac{1}{x} \sum_{n \leqslant x} f(n)^{2}-M^{2}\right\} \\
& =\frac{4}{M^{2}}\left\{M_{2}-M^{2}\right\}, \text { say. }
\end{aligned}
$$

In view of (3), (6), and (5) (with a sufficiently large constant $C$ ), the asserted upper bound (4) follows if we can show

$$
M_{2}=\lim _{x \rightarrow \infty} \frac{1}{x} \sum_{n \leqslant x} f(n)^{2} \leqslant\left(\frac{\log \log D}{\varphi(r)}\right)^{2}+O\left(\frac{\log \log D}{\varphi(r)}\right)
$$

with an absolute $O$-constant.

Expanding $f(n)^{2}$, we get

$$
M_{2}=\sum_{d, d^{\prime} \in \mathscr{D}} \frac{1}{\left[d, d^{\prime}\right]} \lim _{x \rightarrow \infty} \frac{\left[d, d^{\prime}\right]}{x} \sum_{\substack{n \leqslant x /\left[d, d^{\prime}\right] \\(*)}} 1,
$$

where $(*)$ denotes the condition

$$
\frac{n d}{\left(d, d^{\prime}\right)} \equiv \frac{n d^{\prime}}{\left(d, d^{\prime}\right)} \equiv-1 \bmod r .
$$

Let $d=d_{1} p$ and $d^{\prime}=d_{1}^{\prime} p^{\prime}$ be the (unique) decompositions of the form (2) for $d$ and $d^{\prime}$. Then (*) has a solution in $n$ if and only if $d_{1}=d_{1}^{\prime}, p \equiv p^{\prime} \bmod r$, and in this case the limit in the last expression equals $1 / r$. Thus we get

$$
\begin{aligned}
M_{2} & \leqslant \frac{1}{r} \sum_{d_{1} \leqslant D} \frac{1}{d_{1}} \sum_{\substack{p, p^{\prime} \leqslant D \\
p \equiv p^{\prime} \bmod r \\
p+r}} \frac{1}{\left[p, p^{\prime}\right]} \\
& \leqslant \frac{1}{\varphi(r)} \sum_{\substack{p \leqslant D \\
p+r}} \frac{1}{p}\left(\sum_{\substack{p^{\prime} \leqslant D \\
p^{\prime} \equiv p \bmod r}} \frac{1}{p^{\prime}}+O(1)\right) .
\end{aligned}
$$


The innermost sum equals

$$
\begin{aligned}
& \sum_{\substack{e^{r} \leqslant p^{\prime} \leqslant D \\
p^{\prime} \equiv p \bmod r}} \frac{1}{p^{\prime}}+O\left(\sum_{\substack{n \leqslant e^{r} \\
n \equiv p \bmod r}} \frac{1}{n}\right) \\
& \quad=\int_{e^{r}}^{D} \pi(x, r, p) \frac{d x}{x^{2}}+O(1)=\frac{\log \log D}{\varphi(r)}+O(1),
\end{aligned}
$$

where the last step follows from the Siegel-Walfisz theorem. We therefore obtain

$$
\begin{aligned}
M_{2} & \leqslant \frac{1}{\varphi(r)}\left(\sum_{p \leqslant D} \frac{1}{p}\right)\left(\frac{\log \log D}{\varphi(r)}+O(1)\right) \\
& \leqslant\left(\frac{\log \log D}{\varphi(r)}\right)^{2}+O\left(\frac{\log \log D}{\varphi(r)}\right),
\end{aligned}
$$

i.e., estimate (7). This completes the proof of Lemma 2.

4. Proof of the Theorem. For $x>0$ let $\mathrm{d}_{x}(\cdot)$ be defined by

so that

$$
\mathrm{d}_{x}(M)=\frac{1}{x} \sum_{\substack{n \leqslant x \\ n \in M}} 1 \quad(M \subset \mathbb{N})
$$

$$
\mathrm{d}(M)=\liminf _{x \rightarrow \infty} \mathrm{d}_{x}(M), \quad \overline{\mathrm{d}}(M)=\limsup _{x \rightarrow \infty} \mathrm{d}_{x}(M)
$$

If $\lambda \geqslant 1$, then obviously

$$
\mathrm{d}_{x}(\lambda M)=(1 / \lambda) \mathrm{d}_{x / \lambda}(M) \leqslant \mathrm{d}_{x}(M),
$$

and for every fixed $t \in \mathbb{N}$ we have

$$
\mathrm{d}_{x}(M+t)=\mathrm{d}_{x}(M)+o(1) \quad \text { as } x \rightarrow \infty .
$$

Given a set $A \subset \mathbb{N}$ and positive integers $n_{1}<\cdots<n_{k}$ satisfying (1), we define the sets

$$
B_{i, d}=n_{i}(A+d) \cap n d \mathbb{N} \quad(1 \leqslant i \leqslant k, d \in \mathbb{N}),
$$

where $n=\prod_{i=1}^{k} n_{i}^{2}$. By the inclusion-exclusion principle we have, for $x>0$ and every $d \in \mathbb{N}$,

$$
\mathrm{d}_{x}\left(\bigcup_{i=1}^{k} B_{i, d}\right) \geqslant \sum_{i=1}^{k} \mathrm{~d}_{x}\left(B_{i, d}\right)-\sum_{1 \leqslant i<j \leqslant k} \mathrm{~d}_{x}\left(B_{i, d} \cap B_{j, d}\right) .
$$

We shall estimate from above the second term on the right in terms of

$$
\mathrm{d}_{x}\left(A_{N} \cap\left(A_{N}+1\right)\right) \text {, }
$$

where $N \geqslant \max (d, n)$, and bound the first term, averaged over a suitable range for $d$, from below in terms of $\mathrm{d}_{x / n_{k}}(A)$. This will lead to the desired relation between the densities of $A$ and $A_{N} \cap\left(A_{N}+1\right)$.

Using the stated properties of the function $\mathrm{d}_{x}$, we obtain

$$
\begin{aligned}
\mathrm{d}_{x}\left(B_{i, d}\right) & =\mathrm{d}_{x}\left(n_{i}\left((A+d) \cap d \frac{n}{n_{i}} \mathbb{N}\right)\right)=\frac{1}{n_{i}} \mathrm{~d}_{x / n_{i}}\left((A+d) \cap d \frac{n}{n_{i}} \mathbb{N}\right) \\
& \geqslant \frac{1}{n_{k}} \mathrm{~d}_{x / n_{k}}\left(A \cap d T_{i}\right)+o(1)
\end{aligned}
$$


where $T_{i}=\left(n / n_{i}\right) \mathbb{N}-1$. Moreover, for $1 \leqslant i<j \leqslant k$, we get

$$
\begin{aligned}
\mathrm{d}_{x}\left(B_{i, d} \cap B_{j, d}\right) & \leqslant \mathrm{d}_{x}\left(n_{i}(A+d) \cap n_{j}(A+d) \cap d n_{i} n_{j} \mathbb{N}\right) \\
& \leqslant \mathrm{d}_{x}\left(\frac{n_{i}}{\left(n_{i}, n_{j}\right)} \frac{A \cap d \mathbb{N}}{d} \cap\left(\frac{n_{j}}{\left(n_{i}, n_{j}\right)} \frac{A \cap d \mathbb{N}}{d}+\frac{n_{j}-n_{i}}{\left(n_{i}, n_{j}\right)}\right)\right)+o(1) \\
& \leqslant \mathrm{d}_{x}\left(A_{N} \cap\left(A_{N}+1\right)\right)+o(1),
\end{aligned}
$$

provided $N \geqslant \max (n, d)$, where the last step follows from (1) and the definition of $A_{N}$. Substituting these estimates together with the trivial bound

$$
\mathrm{d}_{x}\left(\bigcup_{i=1}^{k} B_{i, d}\right) \leqslant \mathrm{d}_{x}(d n \mathbb{N}) \leqslant \frac{1}{d n}
$$

into (8) yields

$$
\frac{1}{d n} \geqslant \frac{1}{n_{k}} \sum_{i=1}^{k} \mathrm{~d}_{x / n_{k}}\left(A \cap d T_{i}\right)-k^{2} \mathrm{~d}_{x}\left(A_{N} \cap\left(A_{N}+1\right)\right)+o(1)
$$

for every fixed $d \in \mathbb{N}$ and $N \geqslant \max (n, d)$.

We now fix $D \geqslant 1$ and let $\mathscr{D}=\mathscr{D}\left(D, n / n_{i}\right)$ be defined as in Lemma 2 , with $r=n / n_{i}$. Since $\mathscr{D}(D, r)$ depends only on the set of prime factors of $r$, and the numbers $n / n_{i}=\prod_{j=1}^{k} n_{j}^{2} / n_{i}, 1 \leqslant i \leqslant k$, have the same set of prime factors, this definition does not depend on the choice of the index $i$. Summing the last inequality over $d \in \mathscr{D}$, we obtain, for $N \geqslant \max (n, D)$,

$$
\begin{aligned}
\frac{1}{n} \sum_{d \in \mathscr{D}} \frac{1}{d} & \geqslant \frac{1}{n_{k}} \sum_{i=1}^{k} \sum_{d \in \mathscr{D}} \mathrm{d}_{x / n_{k}}\left(A \cap d T_{i}\right)-D k^{2} \mathrm{~d}_{x}\left(A_{N} \cap\left(A_{N}+1\right)\right)+o(1) \\
& \geqslant \frac{1}{n_{k}} \sum_{i=1}^{k} \mathrm{~d}_{x / n_{k}}\left(A \cap S_{i}\right)-D k^{2} \mathrm{~d}_{x}\left(A_{N} \cap\left(A_{N}+1\right)\right)+o(1),
\end{aligned}
$$

where

$$
S_{i}=\bigcup_{d \in \mathscr{D}} d T_{i}=\bigcup_{d \in \mathscr{D}} d\left(\frac{n}{n_{i}} \mathbb{N}-1\right) .
$$

Letting $x \rightarrow \infty$, we deduce

$$
\begin{aligned}
D k^{2} \underline{\mathrm{d}}\left(A_{N} \cap\left(A_{N}+1\right)\right) & \geqslant \frac{1}{n_{k}} \sum_{i=1}^{k} \mathrm{~d}\left(A \cap S_{i}\right)-\frac{1}{n} \sum_{d \in \mathscr{D}} \frac{1}{d} \\
& \geqslant \frac{k}{n_{k}} \underline{\mathrm{d}}(A)-\frac{1}{n_{k}} \sum_{i=1}^{k} \mathrm{~d}\left(\mathbb{N} \backslash S_{i}\right)-\frac{1}{n} \sum_{d \in \mathscr{D}} \frac{1}{d} .
\end{aligned}
$$

By Lemma 2 we have

$$
\frac{n_{k}}{n} \sum_{d \in \mathscr{D}} \frac{1}{d} \ll \frac{\log \log (D+2)}{\varphi\left(n / n_{k}\right)}+1
$$

and

$$
\mathrm{d}\left(\mathbb{N} \backslash S_{i}\right) \ll \frac{\varphi\left(n / n_{i}\right)}{\log \log (D+2)}
$$


Since

$$
\frac{\varphi\left(n / n_{i}\right)}{n / n_{i}}=\prod_{p \mid n / n_{i}}\left(1-\frac{1}{p}\right)=\prod_{p \mid n_{1} \cdots n_{k}}\left(1-\frac{1}{p}\right)
$$

is independent of the choice of $i$, and since, in view of (1),

$$
n_{1} \leqslant n_{i} \leqslant n_{k} \leqslant 2 n_{1},
$$

the last estimate remains valid with $\varphi\left(n / n_{k}\right)$ in place of $\varphi\left(n / n_{i}\right)$. Thus, defining $D=D(k)$ by

$$
\frac{\log \log (D+2)}{\varphi\left(n / n_{k}\right)}=\sqrt{k}
$$

we obtain, from (9),

$$
D k^{2} \underline{\mathrm{d}}\left(A_{N} \cap\left(A_{N}+1\right)\right) \geqslant \frac{k}{n_{k}}\left(\underline{\mathrm{d}}(A)+O\left(\frac{1}{\sqrt{k}}\right)\right)
$$

with an absolute $O$-constant. If now $\mathrm{d}(A) \geqslant \varepsilon(>0)$, then by choosing $k=k(\varepsilon)$ sufficiently large (which is possible by Lemma 1 ), the $O$-term becomes $\leqslant \varepsilon / 2$, and we get

$$
\underline{\mathrm{d}}\left(A_{N} \cap\left(A_{N}+1\right)\right) \geqslant \delta(\varepsilon) \quad(N \geqslant N(\varepsilon)),
$$

with

$$
\delta(\varepsilon)=\frac{\varepsilon}{2 D(k) k n_{k}}, \quad N(\varepsilon)=\max (n, D(k)),
$$

as asserted in the Theorem.

By a minor modification of the proof, one can show that the theorem remains valid, when $\mathrm{d}$ is replaced by the upper density $\overline{\mathrm{d}}$.

ACKNOWLEDGEMENT. This work was done while the author was visiting the University of Illinois at Urbana-Champaign. I would like to thank the Department of Mathematics at Urbana for its hospitality and the Deutsche Forschungsgemeinschaft for financial support. Also, I am grateful to A. Balog for calling my attention to reference [4].

\section{REFERENCES}

1. A. Balog, Problem in Tagungsbericht 41 (1982), Math. Forschungsinstitut Oberwolfach, p. 29.

2. N. G. de Bruijn, On the number of positive integers $\leqslant x$ and free of prime factors $>y$, Nederl. Akad. Wetensch. Proc. Ser. A 54 (1951), 50-60.

3. P. Erdös, Problems and results on number theoretic properties of consecutive integers and related questions, Proc. Fifth Manitoba Conf. on Num. Math. (Univ. Manitoba, Winnipeg, Man., 1975). Congressus Numeratium, no. XVI, Utilitas Math., Winnipeg, Man., 1976, pp. 25-44.

4. D. R. Heath-Brown, The divisor function at consecutive integers, Mathematika 31 (1984), 141-149.

DePartment of Mathematics, University of Illinois, Urbana, Illinois 61801

Current address: School of Mathematics, Institute for Advanced Study, Princeton, New Jersey 08540 